\title{
Metabolic Engineering of Saccharomyces cerevisiae to Improve Glucan Biosynthesis
}

\author{
Xing Zhou ${ }^{1,2}$, Jing $\mathrm{He}^{1,2}$, Lingling Wang ${ }^{1,2}$, Yang Wang ${ }^{1,2}$, Guocheng $\mathrm{Du}^{1,2}$, and Zhen Kang ${ }^{1,2 *}$ \\ ${ }^{1}$ The Key Laboratory of Carbohydrate Chemistry and Biotechnology, Ministry of Education, School of Biotechnology, Jiangnan University, \\ Wuxi 214122, P.R. China \\ ${ }^{2}$ The Key Laboratory of Industrial Biotechnology, Ministry of Education, School of Biotechnology, Jiangnan University, Wuxi 214122, P.R. China
}

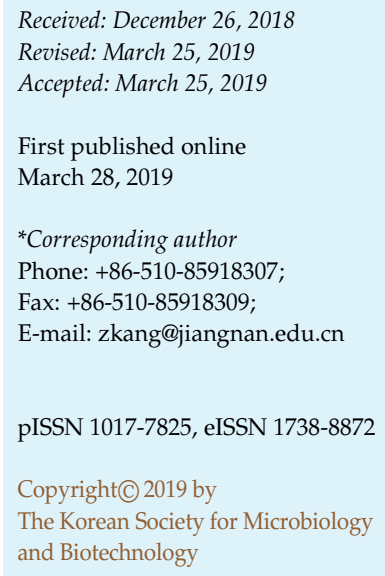

\section{Introduction}

$\beta$-glucan is widely distributed in bacteria, algae, mushrooms, and unicellular fungi such as Saccharomyces cerevisiae and Candida albicans and higher plants [1] as a main constituent of cell wall structure. $\beta$-Glucan has many reported biological functions such as immune modulation and enhancement of resistance against infections and cancer development [2]. Moreover, $\beta$-glucans can also provide protection against oxidation [3] and radiation, and lower the level of cholesterol and blood lipids. Therefore, $\beta$-glucans, especially those extracted from Saccharomyces cerevisiae, have been widely used in the medical, health food and cosmetics industries [4-6].

The cell wall of $S$. cerevisiae consists of a bilayered structure of $\beta-1,3$-glucan, mannoprotein and $\beta-1,6$-glucan $[7,8]$. $\beta-1$, 3 -Glucan and $\beta-1,6$-glucan account for approximately 50 $55 \%$ and $10-15 \%$ of the total cell wall polysaccharide, respectively $[8,9]$. $\beta-1,3-G l u c a n$ as a major constituent forms a network by attaching to the heavily branched $\beta-1$, 6 -glucan $[10,11]$. To date, three membrane-associated $\beta-1$, 3-glucan synthases from $S$. cerevisiae have been identified.
The major $\beta$-1, 3-glucan synthase Fks1 has been well studied [12]. In particular, Fks1 was found in association with Rho1, a GTPase for activating glucan synthesis [13, 14]. In contrast, the $\beta$-1, 6-glucan synthase activity has not been characterized and the regulatory mechanism remains unclear, although many KRE genes, such as KRE5, KRE6, and KRE9 have been repeatedly demonstrated to be involved in $\beta$-1, 6-glucan synthesis [15].

The $\beta$-glucan biosynthesis pathway in $S$. cerevisiae is shown in Fig. 1. After transportation and phosphorylation, glucose-6-phosphate is converted to glucose-1-phosphate by phosphoglucomutase. Then, the polymer precursor UDP-glucose is synthesized from UTP and glucose-1phosphate by UDP-glucose pyrophosphorylase. Finally, the glucan synthases Fks1, Fks2, and Fks3 in association with Rho1 polymerize $\beta$-glucan from UDP-glucose. Although many efficient strategies for extracting $S$. cerevisiae $\beta$-glucan have been developed, no efforts have been spent on strengthening $\beta$-glucan accumulation from the perspective of metabolic pathway engineering. In this study, we engineered the $\beta$-glucan biosynthesis pathway in S. cerevisiae for the first time. 


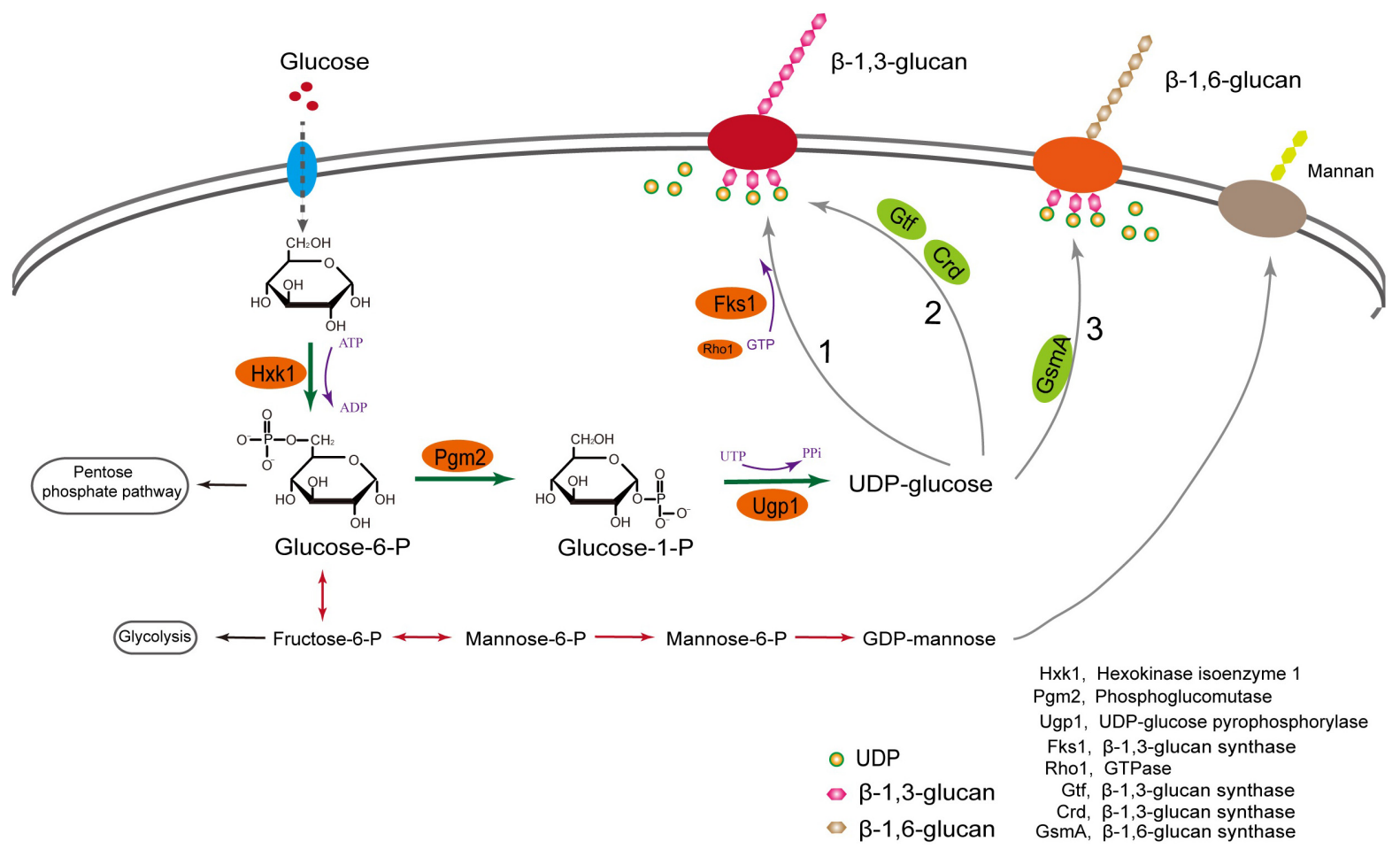

Fig. 1. Biosynthetic pathway of $\beta$-glucan from glucose in S. cerevisiae.

1, Glucan synthases Fks1, Fks2, and Fks3 (only one is shown in the figure) synthesize $\beta$-1, 3-glucan from UDP-glucose; 2, Exogenous $\beta$-1, 3-glucan synthase Gtf, Crd synthesize $\beta$-1, 3-glucan from UDP-glucose; 3, Exogenous $\beta$-1, 6-glucan synthase GsmA synthesizes $\beta$-1, 6-glucan from UDPglucose.

\section{Materials and Methods}

\section{Plasmid and Strain Construction}

All strains and plasmids used in this study are listed in Table 1. Saccharomyces cerevisiae CEN.PK2-1C was used as the wild-type strain. Glucan synthesis pathway genes RHO1, HXK1, PGM2 and UGP1 were amplified from the genome of $S$. cerevisiae S288c with designated oligonucleotides (Table 2). PCR fragments of the five genes were digested with enzymes Bam HI and PstI, and subcloned into YEp351. The genes $g t f, c r d$, and $g s m A$ were synthesized by Genscript (China), and inserted into pRS305 after digestion with enzymes BamHI and PstI. These recombinant plasmids (Table 1) were transformed into S. cerevisiae CEN.PK2-1C by LiAc/SSDNA/ PEG and the transformants were screened on synthetic dropout plate and further confirmed with PCR.

\section{Medium and Culture Conditions}

LB medium (10 g/l tryptone, $5 \mathrm{~g} / 1$ yeast extract and $10 \mathrm{~g} / 1$ $\mathrm{NaCl}, \mathrm{pH}$ 7.0) was used for E. coli cultures and for all DNA manipulations. Ampicillin $(100 \mu \mathrm{g} / \mathrm{ml})$ was added to provide selective pressure during growth when necessary. Recombinant yeast cells were grown in YPD medium (10 g/l yeast extract, $20 \mathrm{~g} / 1$ peptone, $20 \mathrm{~g} / 1$ glucose). Synthetic dropout (SD) medium [0.67\% $(\mathrm{w} / \mathrm{v})$ yeast nitrogen base, $2 \%(\mathrm{w} / \mathrm{v})$ glucose] media with appropriate supplemental amino acids were used to select yeast transformants.

\section{Analysis of the Polysaccharides of Different Strains}

The contents of polysaccharides in yeast cell wall were measured to examine the influences of overexpressed genes on yeast cell wall structure. Cells were grown to the early exponential phase $\left(2.5 \times 10^{6}\right.$ cells) and harvested by centrifugation $(10,000 \times g, 1 \mathrm{~min})$. Cells were incubated in $50 \mathrm{ml}$ of $0.1 \mathrm{M}$ acetic acid-sodium acetate (pH 5.0), at $55^{\circ} \mathrm{C}$ for $24 \mathrm{~h}$ to induce cell autolysis. The autolyzed cells were further lysed by cryogenic grinding. The isolated cell wall was treated with $72 \%(\mathrm{v} / \mathrm{v}) \mathrm{H}_{2} \mathrm{SO}_{4}$ at room temperature for $4 \mathrm{~h}$ and hydrolyzed with $2 \mathrm{M} \mathrm{H}_{2} \mathrm{SO}_{4}$ at $100^{\circ} \mathrm{C}$ for $4 \mathrm{~h}$. After adjusting $\mathrm{pH}$ to 7.0 , the composition of monosaccharides (mannose and glucose) was analyzed and quantified with high-performance anionic exchange chromatography. The content of polysaccharides in the cell wall of strains was calculated according to the following equation:

Glucan content $=\mathrm{M}_{\text {(polysaccharide) }} / \mathrm{CDW}$,

where $\mathrm{M}_{\text {(polysaccharide) }}$ is the amount (mg) of polysaccharides while cell dry weight (CDW) is expressed in $\mathrm{g}$. 
Table 1. Plasmids and strains used in this study.

\begin{tabular}{|c|c|c|}
\hline Plasmids and strains & Genotype & References \\
\hline \multicolumn{3}{|l|}{ Plasmids } \\
\hline pRS305 & E. coli/S. cerevisiae shuttle vector, ScLEU2, $2 \mu \mathrm{m}, \mathrm{Ap}^{\mathrm{r}}$ & [29] \\
\hline pRS305-gtf & $P_{T E F}-g t f-T_{C Y C 1}$ in pRS305 & This work \\
\hline pRS305-crd & $P_{T E F}-c r d-T_{C Y C 1}$ in pRS305 & This work \\
\hline pRS305-gsmA & $P_{T E F}-g S m A-T_{C Y C 1}$ in pRS305 & This work \\
\hline pY26 & E. coli/S. cerevisiae shuttle vector, ScURA3, $2 \mu \mathrm{m}, \mathrm{Ap}^{\mathrm{r}}$ & This work \\
\hline pY26-RHO1 & $P_{G A P^{-}}$RHO1- $T_{C Y C 1}$ in YEp351 & This work \\
\hline pY26-HXK1 & $P_{G A P}-H X K 1-T_{C Y C 1}$ in YEp351 & This work \\
\hline pY26-PGM2 & $P_{G A P^{-}} P G M 2-T_{C Y C 1}$ in YEp351 & This work \\
\hline pY26-UGP1 & $P_{G A P^{-}}$UGP1-T $T_{C Y C 1}$ in YEp351 & This work \\
\hline \multicolumn{3}{|l|}{ Strains } \\
\hline E. coli JM109 & $\begin{array}{l}F^{\prime} \text { traD36 proA } A^{+} B^{+} \text {lacIq } \Delta(\text { lacZ) M15/A(lac-proAB) } \\
\text { glnV44e14- gyrA96 recA1 relA1 endA1 thi hsdR17 }\end{array}$ & Invitrogen \\
\hline S. cerevisiae & MATa; ura3-52; $\operatorname{trp1-289;~leu2-3,112;~his3?~1;~MAL2-8c;~}$ & Lab stock \\
\hline CEN.PK2-1C & SUC2 & This work \\
\hline S1 & S. cerevisiae CEN.PK2-1C/ pRS305 & This work \\
\hline S2 & S. cerevisiae CEN.PK2-1C/ pY26 & This work \\
\hline S3 & S. cerevisiae CEN.PK2-1C/ pRS305, pY26 & This work \\
\hline S-gtf & S. cerevisiae CEN.PK2-1C/ pRS305-gtf & This work \\
\hline S-crd & S. cerevisiae CEN.PK2-1C/ pRS305-crd & This work \\
\hline S-gsmA & S. cerevisiae CEN.PK2-1C/ pRS305-gsmA & This work \\
\hline S-HXK1 & S. cerevisiae CEN.PK2-1C/ pY26-HXK1 & This work \\
\hline S-PGM2 & S. cerevisiae CEN.PK2-1C/ pY26-PGM2 & This work \\
\hline S-UGP1 & S. cerevisiae CEN.PK2-1C/ pY26-UGP1 & This work \\
\hline $\mathrm{S}-\mathrm{RHO1}$ & S. cerevisiae CEN.PK2-1C/ pY26-RHO1 & This work \\
\hline G-pY26 & S. cerevisiae CEN.PK2-1C/ gsmA, pY26 & This work \\
\hline G-HXK1 & S. cerevisiae CEN.PK2-1C/ gsmA, pY26-HXK1 & This work \\
\hline G-PGM2 & S. cerevisiae CEN.PK2-1C/ gsmA, pY26-PGM2 & This work \\
\hline G-UGP1 & S. cerevisiae CEN.PK2-1C/ gsmA, pY26-UGP1 & This work \\
\hline G-RHO1 & S. cerevisiae CEN.PK2-1C/ gsmA, pY26-RHO1 & This work \\
\hline
\end{tabular}

\section{Transmission Electron Microscopy (TEM) Analysis}

The cell wall structure of yeast strains was analyzed with transmission electron microscopy (TEM). Yeast cells were cultured on plate at $30^{\circ} \mathrm{C}$ for $24 \mathrm{~h}$ and then harvested for TEM analysis. Yeast cell samples were fixed as described [16]. The samples were fixed in $2.5 \%(\mathrm{w} / \mathrm{v})$ glutaraldehyde-PBS buffer $(\mathrm{pH}$ 7.2) overnight at $4{ }^{\circ} \mathrm{C}$, and then rinsed with the same buffer for six times and post-fixed with $1 \%(\mathrm{w} / \mathrm{v})$ osmium tetroxide for $2 \mathrm{~h}$ at $4{ }^{\circ} \mathrm{C}$. The samples were dehydrated stepwise with $30 \%, 50 \%, 70 \%$ and $90 \%(\mathrm{v} / \mathrm{v})$ of ethanol, 1: 1 mixture of $90 \%(\mathrm{v} / \mathrm{v})$ ethanol and $90 \%(\mathrm{v} / \mathrm{v})$ acetone, and acetone for three times. The samples were then embedded in Epon812 and polymerized. Ultrathin sections of the samples were prepared and examined with a transmission electron microscope.

\section{Stress Resistance Assays}

The resistances of mutants to different stresses were tested on SD plate contain $1 \mathrm{M}$ sorbitol and $5 \%(\mathrm{w} / \mathrm{v}) \mathrm{NaCl}$, respectively. Yeast cells were grown in $5 \mathrm{ml} \mathrm{SD}$ at $30^{\circ} \mathrm{C}$ for $24 \mathrm{~h}$ and harvested. Cells were washed twice with distilled water and suspended with $0.9 \%(\mathrm{w} / \mathrm{v})$ saline. Finally, a 10 -fold serial dilution of this culture was made and a $5-\mu \mathrm{l}$ aliquot of each dilution was spotted onto appropriate solid medium and incubated at $30^{\circ} \mathrm{C}$ for 3 days.

\section{Results and Discussion}

\section{Overexpression of Heterologous $\beta$-Glucan Synthases to Increase $\beta$-Glucan Biosynthesis}

The expression of $\beta$-glucan synthase and $\beta$-glucan 
Table 2. Primers used in this study.

\begin{tabular}{ll}
\hline Primers & \multicolumn{1}{c}{ Sequences $\left(5^{\prime}-3^{\prime}\right)$} \\
\hline gtf-F & CGGGATCCATGTTGAACGATAACGATTCTG \\
gtf-R & AACTGCAGTTAATCGTTCCAATCAACAGTCTCAG \\
crd-F & CGGGATCCATGTACTTTCTGCTGAAGGAG \\
crd-R & AACTGCAGTTAACCGAAAGCTCTAGCCAAC \\
gsmA-F & CGGGATCCATGAAGAAAAAACAGAACGTTTTCATCTTCGTG \\
gsmA-R & AACTGCAGTTATTTCTGCTGCGCGC \\
RHO1-F & CGGGATCCATGTCACAACAAGTTGG \\
RHO1-R & AACTGCAGCTATAACAAGACACACTTC \\
HXK1-F & CGGGATCCATGGTTCATTTAGGTCCAAAGAAACCACAGGC \\
HXK1-R & AACTGCAGTTAAGCGCCAATGATACCAAGAGAC \\
PGM2-F & CGGGATCCATGTCATTTCAAATTGAAACGGTTCCCACC \\
PGM2-R & AACTGCAGTTAAGTACGAACCGTTGGTTCTTCAGTTCC \\
UGP1-F & CGGGATCCATGTCCACTAAGAAGCACACC \\
UGP1-R & AACTGCAGTCAATGTTCCAAGATTTGC
\end{tabular}

Bold letters represent the restriction enzyme sites.

biosynthesis in S. cerevisiae are strictly regulated and influenced by environmental conditions [17]. In order to increase the overall polymerization capability, three heterologous $\beta$-glucan synthases, GsmA, Gtf and CrdS, from Mycoplasma agalactiae 5632 [18], Oenococcus oeni [19] and Agrobacterium sp. [20], were overexpressed in S. cerevisiae and the effects on the composition of cell wall polysaccharides were investigated. As shown in Fig. 2A, all recombinant strains accumulated more $\beta$-glucan than the wild-type strain. In particular, overexpression of GsmA resulted in
$43 \%$ increase of $\beta$-glucan content. The result here confirmed that GsmA from M. agalactiae is a functional $\beta-1$, 6-glucan synthase [18]. Moreover, the results demonstrate that introduction of heterologous $\beta-1,3$-glucan synthases can also strengthen the biosynthesis of $\beta-1,3$-glucan in $S$. cerevisiae. Although many KRE genes have been reported to participate in $\beta-1,6$-glucan biosynthesis in S. cerevisiae, no specific $\beta-1,6$-glucan synthase has been determined experimentally [9]. Here, functional expression of the M. agalactiae $\beta-1,6$-glucan synthase GsmA in S. cerevisiae
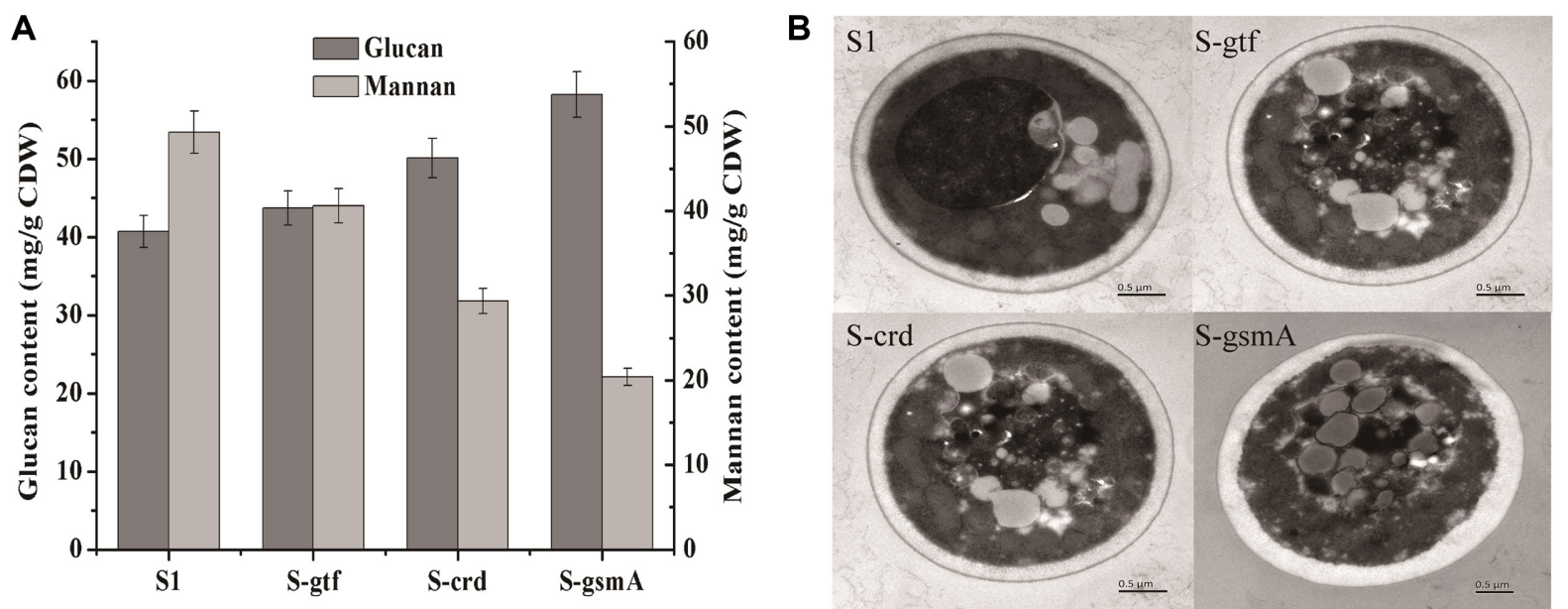

Fig. 2. (A) The content of polysaccharides in the cell wall of strains S1, S-gtf, S-crd, S-gsmA. Yeast cells were cultured in YPD medium. The data shown are mean values from triplicates with error bars indicating the standard deviation; (B) TEM photographs of S. cerevisiae strains S1, S-gtf, S-crd, S-gsmA. 

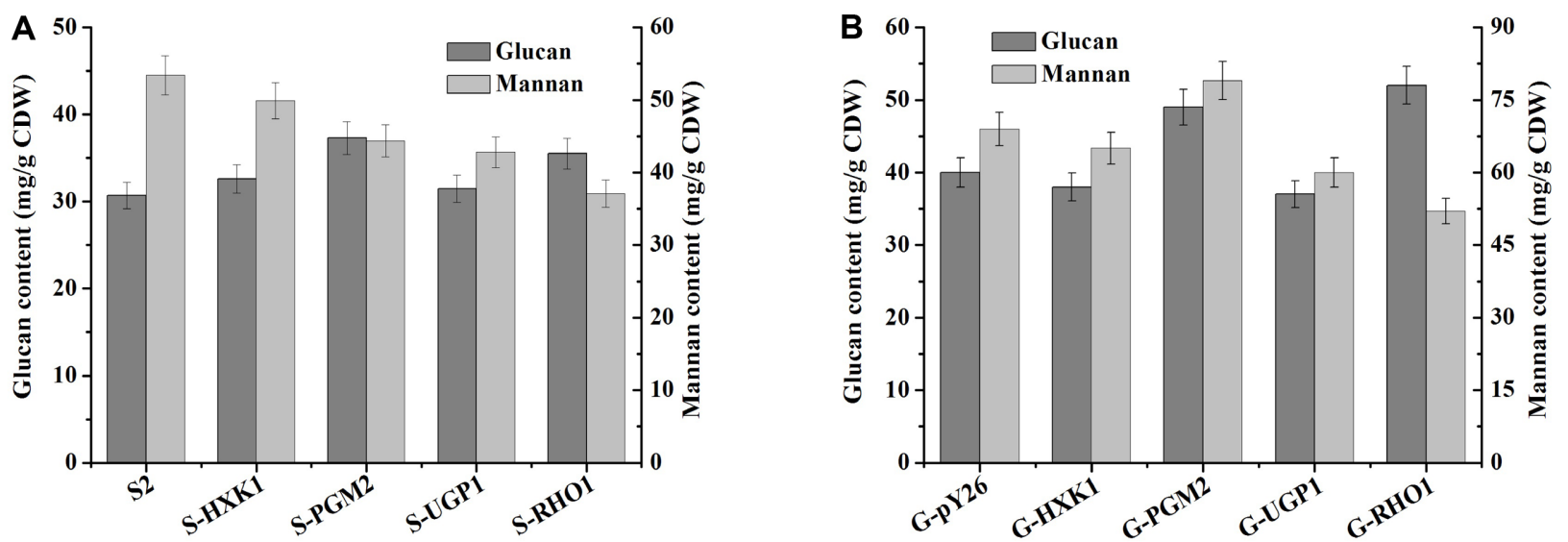

Fig. 3. (A) The content of polysaccharides in the cell wall of strains S2, S-HXK1, S-PGM2, S-UGP1, S-RHO1; (B) The contents of polysaccharides in the cell wall of strains G-pY26, G-HXK1, G-PGM2, G-UGP1, G-RHO1.

Yeast cells were cultured in SD medium. The data shown are mean values from triplicates with error bars indicating the standard deviation.

could help characterize the specific function of the KRE proteins. In bacteria, $\beta-1,3$-glucan is synthesized and secreted in the medium [20]. However, no $\beta$-glucan was detected in the culture medium of $S$. cerevisiae (data not shown). The results suggested that all the produced $\beta-1,6-$ glucan and $\beta-1,3$-glucan by the overexpression of $\beta$-glucan synthases were used as building blocks for cell wall construction.

In addition to $\beta$-glucan, the mannan content of the recombinant S. cerevisiae strains S-gtf, S-crd and S-gsmA was also investigated. Interestingly, the mannan content decreased after the overexpression of heterologous $\beta$-glucan synthases, suggesting competition for substrates (such as glucose-6phosphate) might be ascribed to biosynthesis of $\beta$-glucan and mannan in S. cerevisiae. To observe the change in cell wall structure, TEM analysis was also carried out. According to previous studies, the electron-transparent inner layer is mainly composed of $\beta$-glucan while the electron-dense outer layer consists of mannoprotein [14]. Consistently, overexpression of $\beta$-glucan synthases, especially S-gsmA, increased the thickness of the electron-transparent inner layer (Fig. 2B), suggesting an increased accumulation of $\beta$-glucan.

\section{Optimization of $\beta$-Glucan Biosynthesis Pathway to Enhance Glucan Production}

To direct more UDP-glucose towards $\beta$-glucan biosynthesis, the pathway enzymes Hxk1, Pgm2, Ugp1 and guanosine triphosphatase (Rho1) were separately overexpressed in S. cerevisiae. As shown in Fig. 3A, upregulation of PGM2 and RHO1 increased $\beta$-glucan to $37.3 \mathrm{mg} / \mathrm{g} C D W$ and $35.5 \mathrm{mg} / \mathrm{g} C D W$, respectively. In contrast, no obvious effect was observed when overexpressing HXK1 and UGP1. Similar results were also observed when overexpressing
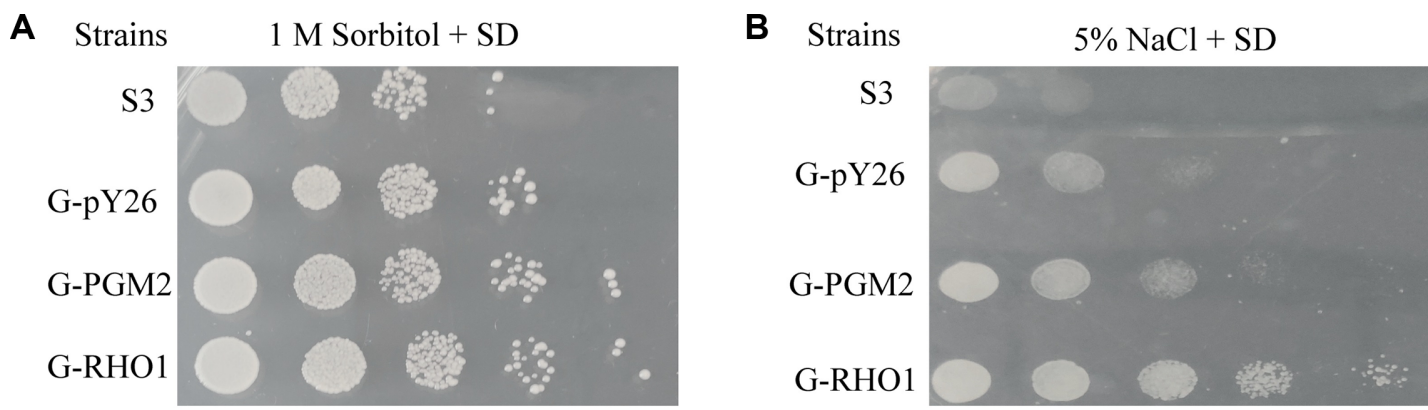

Fig. 4. Assay for sensitivities to different stresses.

(A) Resistance of strains S3, G-pY26, G-PGM2, G-RHO1 to sorbitol; (B) Resistance of strains S3, G-pY26, G-PGM2, G-RHO1 to NaCl. Aliquots (5 $\mu$ l) of 10 -fold serial dilutions of mutant strains and parental strain cells were plated onto the designed plates. 


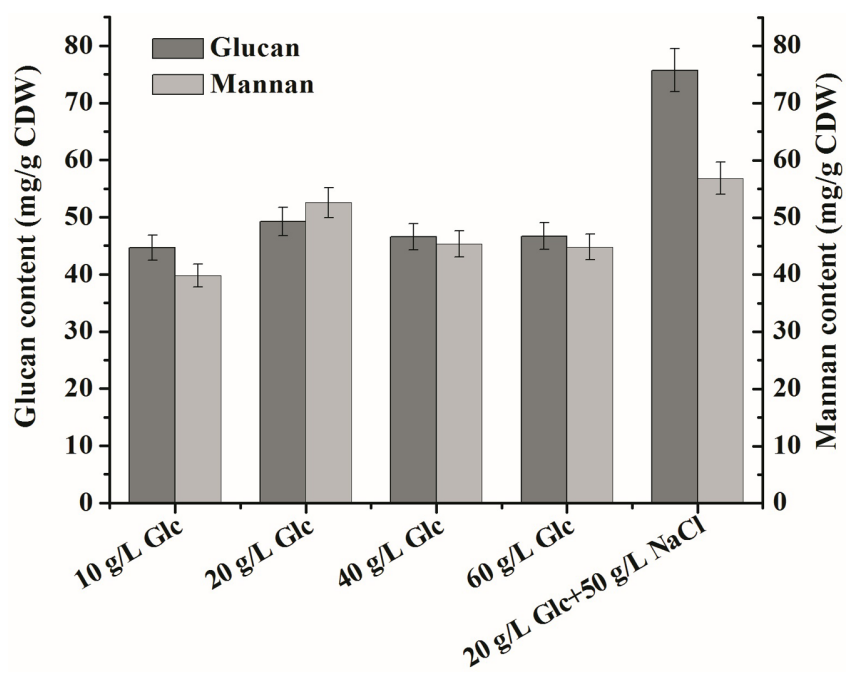

Fig. 5. The content of polysaccharides in the cell wall of strain G-RHO1.

Cells were cultured in SD medium with 10, 20, 40, and $60 \mathrm{~g} / 1$ glucose and $50 \mathrm{~g} / 1 \mathrm{NaCl}$ respectively. The data shown are mean values from triplicates with error bars indicating the standard deviation.

these enzymes in recombinant strain S-gsmA (Fig. 3B). In fact, it has been reported that Pgm2 is a rate-limiting enzyme [21] and its overexpression can significantly improve galactose metabolism in S. cerevisiae [22-24]. Moreover, previous studies have also demonstrated that Rho1 acts as molecular switches to multiple stress conditions [25] and is involved in regulation of cell wall biogenesis in S. cerevisiae [13, 14]. Thus, our results here confirmed that Pgm2 and Rho1 are the committed enzymes for $\beta$-glucan biosynthesis. Furthermore, the resistance ability of the recombinant strains to osmotic stress was increased with $\beta$-glucan accumulation (Fig. 4), demonstrating the significant role of $\beta$-glucan in resistance of yeast cells to environmental stresses.

\section{Optimization of Culture Medium to Increase $\beta$-Glucan Biosynthesis}

The medium composition affects cell growth and generates significant effects on the cell wall structure. Moreover, when encountering environmental stresses, the cell wall remodels into a highly regulated and polarized morphology [13]. For this reason, different concentrations of glucose and $\mathrm{NaCl}$ (high osmotic pressure) were added and the effects on $\beta$-glucan biosynthesis were analyzed. As shown in Fig. 5, no obvious effects were detected when adding different concentrations of glucose. The results indicate that under normal conditions, the $\beta$-glucan content should be constant even in the presence of excess glucose. In contrast, when supplementing $50 \mathrm{~g} / 1 \mathrm{NaCl}$ in $\mathrm{SD}$ medium, the $\beta$-glucan content was increased by $53.1 \%$ while no obvious increase was found for mannan. The results suggest that when yeast cells encounter high osmotic pressure, the $\beta$-glucan biosynthesis is strengthened and as a result, higher content of $\beta$-glucan can be found responsible for cell wall integrity [13].

\section{Acknowledgements}

This work was financially supported by the National Natural Science Foundation of China (31670092), the Fundamental Research Funds for the Central Universities (JUSRP51707A), the National First-class Discipline Program of Light Industry Technology and Engineering (LITE201816), and the Program for Changjiang Scholars and Innovative Research Team in University (grant No. IRT_15R26) and the 111 Project.

\section{Conflict of Interest}

The authors have no financial conflicts of interest to declare.

\section{References}

1. Barsanti L, Passarelli V, Evangelista V, Frassanito AM, Gualtieri P. 2011. Chemistry, physico-chemistry and applications linked to biological activities of beta-glucans. Nat. Prod. Rep. 28: 457-466.

2. Samuelsen ABC, Jürgen S, Knutsen SH. 2014. Effects of orally administered yeast-derived beta-glucans: a review. Mol. Nutr. Food Res. 58: 183-193.

3. Kayali H, Ozdag MF, Kahraman S, Aydin A, Gonul E, Sayal A, et al. 2005. The antioxidant effect of $\beta$-glucan on oxidative stress status in experimental spinal cord injury in rats. Neurosurg. Rev. 28: 298-302.

4. Du B, Bian Z, Xu B. 2014. Skin health promotion effects of natural beta-glucan derived from cereals and microorganisms: a review. Phytother. Res. 28: 159-166.

5. Dalonso N, Goldman GH, Gern RMM. 2015. $\beta-(1 \rightarrow 3),(1 \rightarrow 6)-$ Glucans: medicinal activities, characterization, biosynthesis and new horizons. Appl. Microbiol. Biotechnol. 99: 7893-7906.

6. Rieder A, Ballance S, Böcker U, Knutsen S. 2017. Quantification of 1,3- $\beta$-D-glucan from yeast added as a functional ingredient to bread. Carbohydr. Polym. 181: 34.

7. Yamaguchi M, Namiki Y, Okada H, Mori Y, Furukawa H, Wang J, et al. 2011. Structome of Saccharomyces cerevisiae determined by freeze-substitution and serial ultrathinsectioning electron microscopy. J. Electron Microsc. 60: 321. 
8. Teparić R, Mrsa V. 2013. Proteins involved in building, maintaining and remodeling of yeast cell walls. Curr. Genet. 59: 171-185.

9. Aimanianda V, Clavaud C, Simenel C, Fontaine T, Delepierre M, Latge JP. 2009. Cell wall beta-(1,6)-glucan of Saccharomyces cerevisiae: structural characterization and in situ synthesis. J. Biol. Chem. 284: 13401-13412.

10. Lesage G, Bussey H. 2006. Cell wall assembly in Saccharomyces cerevisiae. Microbiol. Mol. Biol. Rev. 70: 317-343.

11. Latge JP. 2007. The cell wall: a carbohydrate armour for the fungal cell. Mol. Microbiol. 66: 279-290.

12. Klis FM, Boorsma A, De Groot PW. 2006. Cell wall construction in Saccharomyces cerevisiae. Yeast 23: 185-202.

13. Levin DE. 2011. Regulation of cell wall biogenesis in Saccharomyces cerevisiae: the cell wall integrity signaling pathway. Genetics 189: 1145-1175.

14. Xu S, Zhang GY, Zhang H, Kitajima T, Nakanishi H, Gao XD. 2016. Effects of Rho1, a small GTPase on the production of recombinant glycoproteins in Saccharomyces cerevisiae. Microb. Cell Fact. 15: 179.

15. Chavan M, Suzuki T, Rekowicz M, Lennarz W. 2003. Genetic, biochemical, and morphological evidence for the involvement of $\mathrm{N}$-glycosylation in biosynthesis of the cell wall beta1,6-glucan of Saccharomyces cerevisiae. Proc.Natl. Acad. Sci. USA 100: 15381-15386.

16. Guan B, Lei J, Su S, Chen F, Duan Z, Chen Y, et al. 2012. Absence of Yps7p, a putative glycosylphosphatidylinositollinked aspartyl protease in Pichia pastoris, results in aberrant cell wall composition and increased osmotic stress resistance. FEMS Yeast Res. 12: 969-979.

17. Wang J, Li M, Zheng F, Niu C, Liu C, Li Q, et al. 2018. Cell wall polysaccharides: before and after autolysis of brewer's yeast. World J. Microbiol. Biotechnol. 34: 137.

18. Gaurivaud P, Baranowski E, PauRoblot C, Sagné E, Citti C, Tardy F. 2016. Mycoplasma agalactiae secretion of $\beta-(1 \rightarrow 6)-$ glucan, a rare polysaccharide in prokaryotes, is governed by high-frequency phase variation. Appl. Environ. Microbiol. 82: AEM.00274-00216.

19. Dimopoulou M, Vuillemin M, Campbell-Sills H, Lucas PM, Ballestra P, Miot-Sertier C, et al. 2014. Exopolysaccharide
(EPS) synthesis by Oenococcus oeni: from genes to phenotypes. PLoS One 9: e98898.

20. Hrmova M, Stone BA, Fincher GB. 2010. High-yield production, refolding and a molecular modelling of the catalytic module of (1,3)- $\beta$-d-glucan (curdlan) synthase from Agrobacterium sp. Glycoconj. J. 27: 461-476.

21. Liu JJ, Zhang GC, Kong, II, Yun EJ, Zheng JQ, Kweon DH, et al. 2018. A mutation in PGM2 causing inefficient galactose metabolism in the probiotic yeast Saccharomyces boulardii. Appl. Environ. Microbiol. 84(10): pii: e02858-17.

22. Bro C, Knudsen S, Regenberg B, Olsson L, Nielsen J. 2005. Improvement of galactose uptake in Saccharomyces cerevisiae through overexpression of phosphoglucomutase: example of transcript analysis as a tool in inverse metabolic engineering. Appl. Environ. Microbiol. 71: 6465-6472.

23. Garcia Sanchez R, Hahn-Hägerdal B, Gorwa-Grauslund MF. 2010. PGM2 overexpression improves anaerobic galactose fermentation in Saccharomyces cerevisiae. Microb. Cell Fact. 9: 40.

24. Lee KS, Hong ME, Jung SC, Ha SJ, Yu BJ, Koo HM, et al. 2011. Improved galactose fermentation of Saccharomyces cerevisiae through inverse metabolic engineering. Biotechnol. Bioeng. 108: 621-631.

25. Eitzen G, Logan MR. 2012. Analysis of Rho GTPase activation in Saccharomyces cerevisiae. Methods Mol. Biol. 827: 369-380.

26. Auesukaree C. 2017. Molecular mechanisms of the yeast adaptive response and tolerance to stresses encountered during ethanol fermentation. J. Biosci. Bioeng. 124: 133-142.

27. Bleoanca I, Silva AR, Pimentel C, Rodrigues-Pousada C, Menezes Rde A. 2013. Relationship between ethanol and oxidative stress in laboratory and brewing yeast strains. $J$. Biosci. Bioeng. 116: 697-705.

28. Gibson BR, Lawrence SJ, Leclaire JP, Powell CD, Smart KA. 2007. Yeast responses to stresses associated with industrial brewery handling. FEMS Microbiol. Rev. 31: 535-569.

29. Sikorski RS, Hieter P. 1989. A system of shuttle vectors and yeast host strains designed for efficient manipulation of DNA in Saccharomyces cerevisiae. Genetics 122: 19-27. 